\title{
PERTURBATION THEORY FOR THE LU AND QR FACTORIZATIONS
}

\author{
CHI-YE WU ${ }^{1}$ and TING-ZHU HUANG ${ }^{\otimes 2}$
}

(Received 12 March, 2008; revised 27 April, 2008)

\begin{abstract}
In this paper we derive perturbation theorems for the $\mathrm{LU}$ and $\mathrm{QR}$ factors. Moreover, bounds for $\kappa_{L}(A) / \kappa_{L}^{\prime}(A)$ and $\kappa_{U}(A) / \kappa_{U}^{\prime}(A)$ are given for the LU factorization of a nonsingular matrix. By applying pivoting strategies in the $\mathrm{LU}$ factorization, estimates for $\kappa_{L}(P A Q) / \kappa_{L}^{\prime}(P A Q)$ and $\kappa_{U}(P A Q) / \kappa_{U}^{\prime}(P A Q)$ are also obtained.

2000 Mathematics subject classification: 15A23, 65F35.

Keywords and phrases: LU factorization, QR factorization, perturbation.
\end{abstract}

\section{Introduction}

Let $A \in \mathbb{R}^{n \times n}$ be a nonsingular matrix, and suppose that the leading principal submatrices of $A$ are nonsingular. Then $A$ has an LU factorization

$$
A=L U,
$$

where the factors $L$ and $U$ are unit lower triangular and upper triangular matrices, respectively; this factorization is unique (see Higham [5] for details).

Error bounds were derived by Stewart [7] for a first-order expansion of the LU factorization of a perturbation of the identity. He further applied these results to obtain perturbation expansions of the LU, Cholesky and QR factorizations.

Suppose that $A=L^{H} L$ and $A+E=(L+G)^{H}(L+G)$ are the Cholesky factorizations of $A$ and $A+E$, respectively. Sun [9] presented lower and upper bounds for $\|G\| /\|L\|$ in terms of $\|E\| /\|A\|$. Perturbation bounds were also given for the QR factorization of a complex $m \times n$ matrix $A$ of rank $n$. Indefinite $\mathrm{QR}$ factorization is a generalization of the well-known $\mathrm{QR}$ factorization, where $Q$ is a unitary matrix with respect to the given indefinite inner product matrix $J$. Perturbation bounds for

\footnotetext{
${ }^{1}$ School of Applied Mathematics, University of Electronic Science and Technology of China, Chengdu, Sichuan 610054, China.

${ }^{2}$ School of Applied Mathematics, University of Electronic Science and Technology of China, Chengdu, Sichuan 610054, China; e-mail: tingzhuhuang@126.com, tzhuang@uestc.edu.cn.

(C) Australian Mathematical Society 2008, Serial-fee code 0334-2700/08
} 
the so-called "triangular" case of the indefinite QR factorization were given by Sanja Singer and Saša Singer [6].

Chang et al. [3] gave new first-order perturbation bounds for $Q_{1}$ and $R$ in the QR factorization for a given real $m \times n$ matrix of rank $n$; these bounds are generally sharper than the equivalent results for the $R$ factor in [7, 9], and are more straightforward than the sharp result in [10] for the $Q_{1}$ factor alone. Stewart [8] generalized the results mentioned in [3] via a different approach. In this paper, by applying different tools and methods from those used in [8], we derive different perturbation results concerning the factors of $\mathrm{LU}$ decomposition for a given real nonsingular $n \times n$ matrix; moreover, by a similar method, a perturbation theorem for the QR factorization is also obtained.

Chang and Paige [2], on the other hand, studied the sensitivity of the LU factorization; they wanted to investigate $\kappa_{L}(A) / \kappa_{L}^{\prime}(A)$ and $\kappa_{U}(A) / \kappa_{U}^{\prime}(A)$, but results on these ratios did not appear in their earlier work. In this paper we shall fill this gap, and also analyse the ratios of $\kappa_{L}(P A Q) / \kappa_{L}^{\prime}(P A Q)$ and $\kappa_{U}(P A Q) / \kappa_{U}^{\prime}(P A Q)$.

First-order perturbation bounds are frequently used. Dopico and Molera [4] presented expressions for terms of any order in the series expansions of the perturbed LU and Cholesky factors. In this paper, by using the first-order terms in the Taylor series for the $\mathrm{LU}$ and $\mathrm{QR}$ factors of the perturbed matrix $A+t G$, and applying the so-called matrix-vector equation approach, perturbation theorems for the factors are obtained. It is notable that the conditions and results shown in this paper differ from those in [4].

The main purpose of this paper is to establish perturbation theorems for the LU and QR factors and to bound the ratios $\kappa_{L}(A) / \kappa_{L}^{\prime}(A)$ and $\kappa_{U}(A) / \kappa_{U}^{\prime}(A)$. Moreover, by applying pivoting strategies in the computation, we also provide estimates for the ratios $\kappa_{L}(P A Q) / \kappa_{L}^{\prime}(P A Q)$ and $\kappa_{U}(P A Q) / \kappa_{U}^{\prime}(P A Q)$.

\section{Preliminaries}

Previously, the approach that was most often taken for perturbation analyses of factorizations was what we refer to as the "matrix equation" approach. For the LU factorization, this involves expressing the matrix equation as a matrix-vector equation of the form

$$
W\left[\begin{array}{l}
\operatorname{uvec}(\dot{U}(0)) \\
\operatorname{slvec}(\dot{L}(0))
\end{array}\right]=\operatorname{vec}(G) .
$$

Since the leading $(n-1) \times(n-1)$ block of $U$ is nonsingular, $W$ is also nonsingular and, from (2.1),

$$
\left[\begin{array}{l}
\operatorname{uvec}(\dot{U}(0)) \\
\operatorname{slvec}(\dot{L}(0))
\end{array}\right]=W^{-1} \operatorname{vec}(G) .
$$

Partitioning $W^{-1}$ into two blocks, it follows that

$$
W^{-1}=\left[\begin{array}{l}
Y_{U} \\
Y_{L}
\end{array}\right] \text {. }
$$


Then, the condition numbers for the factors $L$ and $U$, defined respectively by

$$
\kappa_{L}(A)=\lim _{\epsilon \rightarrow 0} \sup _{\Delta A}\left\{\frac{\|\Delta L\|_{F}}{\epsilon\|L\|_{F}}: A+\Delta A=(L+\Delta L)(U+\Delta U),\|\Delta A\|_{F} \leq \epsilon\|A\|_{F}\right\}
$$

and

$$
\kappa_{U}(A)=\lim _{\epsilon \rightarrow 0} \sup _{\Delta A}\left\{\frac{\|\Delta U\|_{F}}{\epsilon\|U\|_{F}}: A+\Delta A=(L+\Delta L)(U+\Delta U),\|\Delta A\|_{F} \leq \epsilon\|A\|_{F}\right\},
$$

are given by

$$
\kappa_{L}(A)=\frac{\left\|Y_{L}\right\|_{2}\|A\|_{F}}{\|L\|_{F}} \quad \text { and } \quad \kappa_{U}(A)=\frac{\left\|Y_{U}\right\|_{2}\|A\|_{F}}{\|U\|_{F}} .
$$

Let $U_{n-1}$ denote the leading $(n-1) \times(n-1)$ block of $U$. Then

$$
\kappa_{L}^{\prime}(A)=\inf _{D \in D_{n}} \kappa_{L}^{\prime}(A, D) \text { and } \kappa_{U}^{\prime}(A)=\inf _{D \in D_{n}} \kappa_{U}^{\prime}(A, D),
$$

where

$$
\kappa_{L}^{\prime}(A, D)=\kappa_{2}\left(L D^{-1}\right) \frac{\left\|U_{n-1}^{-1}\right\|_{2}\|A\|_{F}}{\|L\|_{F}}, \quad \kappa_{U}^{\prime}(A, D)=\kappa_{2}\left(D^{-1} \tilde{U}\right) \frac{\left\|L^{-1}\right\|_{2}\|A\|_{F}}{\|U\|_{F}},
$$

with $\tilde{U}=U+\left(\alpha-u_{n n}\right) e_{n} e_{n}^{T}$ for some $\alpha \neq 0$.

To simplify the notation in this paper, for any $n \times n$ matrix $X=\left(x_{i j}\right)$ we define the strictly lower triangular matrix and the upper triangular matrix by

$$
\operatorname{slt}(X)=\left(s_{i j}\right), \quad \operatorname{ut}(X)=X-\operatorname{sit}(X), \quad \text { where } s_{i j}= \begin{cases}x_{i j} & \text { if } i>j, \\ 0 & \text { otherwise. }\end{cases}
$$

Therefore

$$
\|\operatorname{slt}(X)\|_{2} \leq\|X\|_{2}, \quad\|\operatorname{ut}(X)\|_{2} \leq 2\|X\|_{2} .
$$

\section{Perturbation theorems for the $L U$ factors}

In this section, we present the results on how $L$ and $U$ change as $A$ changes, and give perturbation theorems for the factors $L$ and $U$.

THEOREM 3.1. Let $A \in \mathbb{R}_{n}^{n \times n}$ have nonsingular leading $k \times k$ principal submatrices for $k=1, \ldots, n-1$, write its $L U$ factorization as $A=L U$, and suppose that $\Delta A \in \mathbb{R}^{n \times n}$ satisfies $\Delta A=\epsilon G$. If $\epsilon$ is small enough so that the first $n-1$ leading principal submatrices of $A+t G$ are nonsingular for all $|t| \leq \epsilon$, then

$$
\begin{gathered}
\bar{L}^{-1} \dot{L}(0)+\dot{U}(0) \bar{U}^{-1}=\bar{L}^{-1} \bar{G} \bar{U}^{-1}, \\
\dot{L}(0)=\bar{L} \operatorname{sit}\left(\bar{L}^{-1} \bar{G} \bar{U}^{-1}\right), \\
\dot{U}(0)=\operatorname{ut}\left(\bar{L}^{-1} \bar{G} \bar{U}^{-1}\right) \bar{U},
\end{gathered}
$$

where $L+\frac{1}{2} \dot{L}(0) \epsilon=\bar{L}, G+O(\epsilon)=\bar{G}$ and $U+\frac{1}{2} \dot{U}(0) \epsilon=\bar{U}$. 
Proof. From the results of [2], $A+t G$ has the unique LU factorization

$$
A+t G=L(t) U(t), \quad|t|<\epsilon .
$$

Since $L(t)$ and $U(t)$ are continuously differentiable for $|t| \leq \epsilon$ by a standard algorithm for LU factorization, they can be expanded as

$$
L(t)=L(0)+\dot{L}(0) t+O\left(t^{2}\right), \quad U(t)=U(0)+\dot{U}(0) t+O\left(t^{2}\right) .
$$

Setting $t=\epsilon$, we write

$$
L(0)=L, \quad U(0)=U, \quad L(\epsilon)=L+\Delta L, \quad U(\epsilon)=U+\Delta U .
$$

Then (3.5) can be rewritten as

$$
L(t)=L+\dot{L}(0) \epsilon+O\left(\epsilon^{2}\right), \quad U(t)=U+\dot{U}(0) \epsilon+O\left(\epsilon^{2}\right) .
$$

Combining (3.4) with (3.6), it follows that

$$
\begin{aligned}
A+\epsilon G & =\left(L+\dot{L}(0) \epsilon+O\left(\epsilon^{2}\right)\right)\left(U+\dot{U}(0) \epsilon+O\left(\epsilon^{2}\right)\right) \\
& =L U+L \dot{U}(0) \epsilon+\dot{L}(0) U \epsilon+\dot{L}(0) \dot{U}(0) \epsilon^{2}+O\left(\epsilon^{2}\right) .
\end{aligned}
$$

From (1.1) and (3.7), we deduce

$$
\begin{aligned}
G & =L \dot{U}(0)+\dot{L}(0) U+\dot{L}(0) \dot{U}(0) \epsilon+O(\epsilon) \\
& =\left(L+\frac{1}{2} \dot{L}(0) \epsilon\right) \dot{U}(0)+\dot{L}(0)\left(U+\frac{1}{2} \dot{U}(0) \epsilon\right)+O(\epsilon) .
\end{aligned}
$$

Multiplying by $\left(L+\frac{1}{2} \dot{L}(0) \epsilon\right)^{-1}$ on the left and by $\left(U+\frac{1}{2} \dot{U}(0) \epsilon\right)^{-1}$ on the right, (3.8) becomes

$$
\begin{aligned}
& \left(L+\frac{1}{2} \dot{L}(0) \epsilon\right)^{-1}(G+O(\epsilon))\left(U+\frac{1}{2} \dot{U}(0) \epsilon\right)^{-1} \\
& \quad=\dot{U}(0)\left(U+\frac{1}{2} \dot{U}(0) \epsilon\right)^{-1}+\left(L+\frac{1}{2} \dot{L}(0) \epsilon\right)^{-1} \dot{L}(0) .
\end{aligned}
$$

Setting

$$
L+\frac{1}{2} \dot{L}(0) \epsilon=\bar{L}, \quad G+O(\epsilon)=\bar{G}, \quad U+\frac{1}{2} \dot{U}(0) \epsilon=\bar{U},
$$

(3.9) can be rearranged to

$$
\bar{L}^{-1} \dot{L}(0)+\dot{U}(0) \bar{U}^{-1}=\bar{L}^{-1} \bar{G} \bar{U}^{-1}
$$

Note that $\bar{L}^{-1} \dot{L}(0)$ is strictly lower triangular and $\dot{U}(0) \bar{U}^{-1}$ is upper triangular; thus we obtain

$$
\bar{L}^{-1} \dot{L}(0)=\operatorname{sit}\left(\bar{L}^{-1} \bar{G} \bar{U}^{-1}\right), \quad \dot{U}(0) \bar{U}^{-1}=\operatorname{ut}\left(\bar{L}^{-1} \bar{G} \bar{U}^{-1}\right) .
$$


Then

$$
\dot{L}(0)=\bar{L} \operatorname{sit}\left(\bar{L}^{-1} \bar{G} \bar{U}^{-1}\right), \quad \dot{U}(0)=\operatorname{ut}\left(\bar{L}^{-1} \bar{G} \bar{U}^{-1}\right) \bar{U} .
$$

Following a different route, Chang and Paige [2] established the equations

$$
\begin{gathered}
L^{-1} \dot{L}(0)+\dot{U}(0) \tilde{U}^{-1}=L^{-1} G \tilde{U}^{-1}, \\
\dot{L}(0)=L \operatorname{sit}\left(L^{-1} G \tilde{U}^{-1}\right), \\
\dot{U}(0)=\operatorname{ut}\left(L^{-1} G \tilde{U}^{-1}\right) \tilde{U},
\end{gathered}
$$

with $\tilde{U}=U+\left(\alpha-u_{n n}\right) e_{n} e_{n}^{T}$ for some $\alpha \neq 0$. The above equations are clearly analogous in form to those in Theorem 3.1.

THEOREM 3.2. Under the same conditions as in Theorem 3.1, further suppose that

$$
\frac{1}{2}\left\|L^{-1} \dot{L}(0)\right\|_{2} \epsilon<1 \quad \text { and } \quad \frac{1}{2}\left\|U^{-1} \dot{U}(0)\right\|_{2} \epsilon<1 .
$$

Then

$$
\begin{gathered}
\frac{\|\dot{L}(0)\|_{2}}{\|L\|_{2}} \leq \frac{\phi+O(\epsilon)}{1-\frac{1}{2}\left(\kappa_{2}(L)+1\right) \phi \epsilon}, \\
\frac{\|\dot{U}(0)\|_{2}}{\|U\|_{2}} \leq \frac{2\left\|U^{-1}\right\|_{2}\left\|L^{-1}\right\|_{2}\|G\|_{2}+O(\epsilon)}{1-\left(\kappa_{2}(U)+1\right)\left\|L^{-1}\right\|_{2}\|G\|_{2}\|\dot{U}(0)\|_{2}\left\|U^{-1}\right\|_{2} \epsilon}
\end{gathered}
$$

where $\phi=\left\|L^{-1}\right\|_{2}\|G\|_{2}\left\|U^{-1}\right\|_{2}$.

PROOF. From the assumptions we obtain

$$
\begin{aligned}
\left(L+\frac{1}{2} \dot{L}(0) \epsilon\right)^{-1} & =\left(I+\frac{1}{2} L^{-1} \dot{L}(0) \epsilon\right)^{-1} L^{-1} \\
& =\left(I-\frac{1}{2} L^{-1} \dot{L}(0) \epsilon+O\left(\epsilon^{2}\right)\right) L^{-1} \\
\left(U+\frac{1}{2} \dot{U}(0) \epsilon\right)^{-1} & =\left(I+\frac{1}{2} U^{-1} \dot{U}(0) \epsilon\right)^{-1} U^{-1} \\
& =\left(I-\frac{1}{2} U^{-1} \dot{U}(0) \epsilon+O\left(\epsilon^{2}\right)\right) U^{-1}
\end{aligned}
$$

It follows from (3.12) and (3.13) that

$$
\begin{aligned}
\bar{L}^{-1} \bar{G} \bar{U}^{-1} \\
\quad=\left(L+\frac{1}{2} \dot{L}(0) \epsilon\right)^{-1}(G+O(\epsilon))\left(U+\frac{1}{2} \dot{U}(0) \epsilon\right)^{-1} \\
=\left(I-\frac{1}{2} L^{-1} \dot{L}(0) \epsilon+O\left(\epsilon^{2}\right)\right) L^{-1}(G+O(\epsilon))\left(I-\frac{1}{2} U^{-1} \dot{U}(0) \epsilon+O\left(\epsilon^{2}\right)\right) U^{-1} \\
=\left(L^{-1} G-\frac{1}{2} L^{-1} \dot{L}(0) L^{-1} G \epsilon+O(\epsilon)\right)\left(I-\frac{1}{2} U^{-1} \dot{U}(0) \epsilon+O\left(\epsilon^{2}\right)\right) U^{-1} \\
=L^{-1} G U^{-1}-\frac{1}{2} L^{-1} G U^{-1} \dot{U}(0) U^{-1} \epsilon-\frac{1}{2} L^{-1} \dot{L}(0) L^{-1} G U^{-1} \epsilon+O(\epsilon) .
\end{aligned}
$$


Taking the 2-norm of (3.2) and (3.14) gives

$$
\begin{aligned}
\|\dot{L}(0)\|_{2} \leq & \|\bar{L}\|_{2}\left\|\bar{L}^{-1} \bar{G} \bar{U}^{-1}\right\|_{2} \\
\leq & \left(\|L\|_{2}+\frac{1}{2}\|\dot{L}(0)\|_{2} \epsilon\right)\left(\phi+\frac{1}{2} \phi\|\dot{U}(0)\|_{2}\left\|U^{-1}\right\|_{2} \epsilon\right. \\
& \left.+\frac{1}{2}\left\|L^{-1}\right\|_{2}\|\dot{L}(0)\|_{2} \phi \epsilon+O(\epsilon)\right) \\
= & \left(\|L\|_{2}+\frac{1}{2}\|\dot{L}(0)\|_{2} \epsilon\right)\left(\phi+\frac{1}{2}\left\|L^{-1}\right\|_{2}\|\dot{L}(0)\|_{2} \phi \epsilon+O(\epsilon)\right) \\
= & \kappa_{2}(L)\|G\|_{2}\left\|U^{-1}\right\|_{2}+\frac{1}{2} \kappa_{2}(L)\|\dot{L}(0)\|_{2} \phi \epsilon \\
& +\frac{1}{2}\|\dot{L}(0)\|_{2} \phi \epsilon+O(\epsilon) .
\end{aligned}
$$

Thus we have (3.10)

On the other hand, taking the 2-norm of (3.3) and (3.14) gives

$$
\begin{aligned}
\|\dot{U}(0)\|_{2} \leq & 2\|\bar{U}\|_{2}\left\|\bar{L}^{-1} \bar{G} \bar{U}^{-1}\right\|_{2} \\
\leq & 2\left(\|U\|_{2}+\frac{1}{2}\|\dot{U}(0)\|_{2} \epsilon\right)\left(\phi+\frac{1}{2} \phi\|\dot{U}(0)\|_{2}\left\|U^{-1}\right\|_{2} \epsilon+O(\epsilon)\right) \\
= & 2 \kappa_{2}(U)\left\|L^{-1}\right\|_{2}\|G\|_{2}+\kappa_{2}(U)\left\|L^{-1}\right\|_{2}\|G\|_{2}\|\dot{U}(0)\|_{2}\left\|U^{-1}\right\|_{2} \epsilon \\
& +\left\|L^{-1}\right\|_{2}\|G\|_{2}\|\dot{U}(0)\|_{2}\left\|U^{-1}\right\|_{2} \epsilon+O(\epsilon) .
\end{aligned}
$$

Thus we have (3.11).

\section{Bounds for $\kappa_{L}(A) / \kappa_{L}^{\prime}(A)$ and $\kappa_{U}(A) / \kappa_{U}^{\prime}(A)$}

Based on the definitions of $\kappa_{L}(A)$ and $\kappa_{U}(A)$ and the results in [7] on perturbation of the LU factorization, we obtain

$$
\begin{aligned}
& \kappa_{L}(A) \leq\left\|L^{-1}\right\|_{2}\left\|U^{-1}\right\|_{2}\|A\|_{F}, \\
& \kappa_{U}(A) \leq\left\|L^{-1}\right\|_{2}\left\|U^{-1}\right\|_{2}\|A\|_{F},
\end{aligned}
$$

with $\|\Delta A\|_{F} \leq \epsilon\|A\|_{F}$. Since $\kappa_{L}(A)$ and $\kappa_{U}(A)$ are the condition numbers for the factors $L$ and $U$, from the definitions of $\kappa_{L}^{\prime}(A)$ and $\kappa_{U}^{\prime}(A)$ we find that

$$
\kappa_{L}(A) \leq \kappa_{L}^{\prime}(A), \quad \kappa_{U}(A) \leq \kappa_{U}^{\prime}(A) .
$$

In the section, we give bounds for the ratios $\kappa_{L}(A) / \kappa_{L}^{\prime}(A)$ and $\kappa_{U}(A) / \kappa_{U}^{\prime}(A)$.

THEOREM 4.1. Under the same conditions as in Theorem 3.1, further suppose that

$$
\left\|L^{-1} G U^{-1}\right\|_{2} \epsilon \leq \frac{1}{4}
$$

and $\|\Delta A\|_{F} \leq \epsilon\|A\|_{F}$. Then

$$
\begin{aligned}
& \frac{1}{\kappa_{2}\left(L D^{-1}\right)} \leq \frac{\kappa_{L}(A)}{\kappa_{L}^{\prime}(A)} \leq \frac{\left\|L^{-1}\right\|_{2}\|L\|_{F}\left\|U^{-1}\right\|_{2}}{\inf _{D \in D_{n}} \kappa_{2}\left(L D^{-1}\right)\left\|U_{n-1}^{-1}\right\|_{2}}, \\
& \frac{1}{\kappa_{2}\left(D^{-1} \tilde{U}\right)} \leq \frac{\kappa_{U}(A)}{\kappa_{U}^{\prime}(A)} \leq \frac{\left\|U^{-1}\right\|_{2}\|U\|_{F}}{\inf _{D \in D_{n}} \kappa_{2}\left(D^{-1} \tilde{U}\right)}
\end{aligned}
$$


PROOF. From the assumptions we obtain (4.1). The results of [2] then imply that

$$
\left\|Y_{L}(A)\right\|_{2} \geq\left\|U_{n-1}^{-1}\right\|_{2}, \quad\left\|Y_{U}(A)\right\|_{2} \geq\left\|L^{-1}\right\|_{2} .
$$

Combining (4.3) with (2.2) gives

$$
\kappa_{L}(A) \geq \frac{\left\|U_{n-1}^{-1}\right\|_{2}\|A\|_{F}}{\|L\|_{F}}, \quad \kappa_{U}(A) \geq \frac{\left\|L^{-1}\right\|_{2}\|A\|_{F}}{\|U\|_{F}} .
$$

From the definitions of $\kappa_{L}^{\prime}(A)$ and $\kappa_{U}^{\prime}(A)$, we have

$$
\kappa_{L}^{\prime}(A) \leq \kappa_{2}\left(L D^{-1}\right) \frac{\left\|U_{n-1}^{-1}\right\|_{2}\|A\|_{F}}{\|L\|_{F}}, \quad \kappa_{U}^{\prime}(A) \leq \kappa_{2}\left(D^{-1} \tilde{U}\right) \frac{\left\|L^{-1}\right\|_{2}\|A\|_{F}}{\|U\|_{F}} .
$$

Then, by combining (4.4) and (4.5),

$$
\begin{aligned}
& \frac{\kappa_{L}(A)}{\kappa_{L}^{\prime}(A)} \geq \frac{\left(\left(\left\|U_{n-1}^{-1}\right\|_{2}\|A\|_{F}\right) /\|L\|_{F}\right)}{\kappa_{2}\left(L D^{-1}\right)\left(\left(\left\|U_{n-1}^{-1}\right\|_{2}\|A\|_{F}\right) /\|L\|_{F}\right)}=\frac{1}{\kappa_{2}\left(L D^{-1}\right)}, \\
& \frac{\kappa_{U}(A)}{\kappa_{U}^{\prime}(A)} \geq \frac{\left(\left(\left\|L^{-1}\right\|_{2}\|A\|_{F}\right) /\|U\|_{F}\right)}{\kappa_{2}\left(D^{-1} \tilde{U}\right)\left(\left(\left\|L^{-1}\right\|_{2}\|A\|_{F}\right) /\|U\|_{F}\right)}=\frac{1}{\kappa_{2}\left(D^{-1} \tilde{U}\right)} .
\end{aligned}
$$

From (4.1) and the definitions of $\kappa_{L}^{\prime}(A)$ and $\kappa_{U}^{\prime}(A)$, we conclude that

$$
\begin{aligned}
\frac{\kappa_{L}(A)}{\kappa_{L}^{\prime}(A)} & \leq \frac{\left\|L^{-1}\right\|_{2}\left\|U^{-1}\right\|_{2}\|A\|_{F}}{\inf _{D \in D_{n}} \kappa_{2}\left(L D^{-1}\right)\left(\left(\left\|U_{n-1}^{-1}\right\|_{2}\|A\|_{F}\right) /\|L\|_{F}\right)} \\
& =\frac{\left\|L^{-1}\right\|_{2}\|L\|_{F}\left\|U^{-1}\right\|_{2}}{\inf _{D \in D_{n}} \kappa_{2}\left(L D^{-1}\right)\left\|U_{n-1}^{-1}\right\|_{2}}, \\
\frac{\kappa_{U}(A)}{\kappa_{U}^{\prime}(A)} & \leq \frac{\left\|L^{-1}\right\|_{2}\left\|U^{-1}\right\|_{2}\|A\|_{F}}{\inf _{D \in D_{n}} \kappa_{2}\left(D^{-1} \tilde{U}\right)\left(\left(\left\|L^{-1}\right\|_{2}\|A\|_{F}\right) /\|U\|_{F}\right)} \\
& =\frac{\left\|U^{-1}\right\|_{2}\|U\|_{F}}{\inf _{D \in D_{n}} \kappa_{2}\left(D^{-1} \tilde{U}\right)} .
\end{aligned}
$$

Based on the results of Theorem 3.1, we can also obtain bounds for $\kappa_{L}(A) / \kappa_{L}^{\prime}(A)$ and $\kappa_{U}(A) / \kappa_{U}^{\prime}(A)$. However, here they become $\kappa_{\bar{L}}(A) / \kappa_{\bar{L}}^{\prime}(A)$ and $\kappa_{\bar{U}}(A) / \kappa_{\bar{U}}^{\prime}(A)$, respectively, where

$$
L+\frac{1}{2} \dot{L}(0) \epsilon=\bar{L}, \quad G+O(\epsilon)=\bar{G} \quad \text { and } \quad U+\frac{1}{2} \dot{U}(0) \epsilon=\bar{U} .
$$

In the future, we would like to investigate these quantities; but we omit the analysis from this paper.

It is well known that standard algorithms for LU factorization without pivoting are not numerically stable. Therefore, it is possible that the application of pivoting 
strategies in the computation will cause $\left\|U_{n-1}^{-1}\right\|_{2} /\|L\|_{F}$ to become larger. We cannot, however, say whether the condition number $\kappa_{L}(P A Q)$ is larger or smaller than $\kappa_{L}(A)$. Moreover, upon applying partial pivoting (with $Q=I$ ), we cannot tell whether $\kappa_{U}(P A)$ is larger or smaller than $\kappa_{U}(A)$. Nevertheless, using complete pivoting strategies in the computation can lead to significant improvements in $\kappa_{U}(P A Q)$ and $\kappa_{U}(A)$. Thus it is necessary that the bounds on $\kappa_{L}(P A Q) / \kappa_{L}^{\prime}(P A Q)$ and $\kappa_{U}(P A Q) / \kappa_{U}^{\prime}(P A Q)$ are further studied; we provide some details in the next theorem.

THEOREM 4.2. If the same conditions hold as in Theorem 4.1, then

$$
\begin{aligned}
\frac{6}{\sqrt{2 n(n+1)\left(4^{n}+6 n-1\right)}} & \leq \frac{\kappa_{L}(P A Q)}{\kappa_{L}^{\prime}(P A Q)} \leq \frac{\left\|L^{-1}\right\|_{2}\left\|U^{-1}\right\|_{2}\|L\|_{F}}{\left\|U_{n-1}^{-1}\right\|_{2}}, \\
\frac{1}{\kappa_{2}(\tilde{U})} & \leq \frac{\kappa_{U}(P A Q)}{\kappa_{U}^{\prime}(P A Q)} \leq\left\|U^{-1}\right\|_{2}\|U\|_{F},
\end{aligned}
$$

where $P$ and $Q$ are permutation matrices.

PROOF. With no loss of generality, we apply partial pivoting strategies (with $Q=I$ ) in the computation. By [5, Lemma 8.6], we have $\left|l_{i j}\right| \leq 1$ and $\left|\left(L^{-1}\right)_{i j}\right| \leq 2^{i-j-1}$ for all $i>j$. Thus

$$
\kappa_{2}(L) \leq \kappa_{F}(L) \leq \frac{\sqrt{2 n(n+1)\left(4^{n}+6 n-1\right)}}{6} .
$$

Setting $D=I$ and combining (4.4) and (4.5) with (4.7), we obtain

$$
\frac{\kappa_{L}(P A Q)}{\kappa_{L}^{\prime}(P A Q)} \geq \frac{6}{\sqrt{2 n(n+1)\left(4^{n}+6 n-1\right)}} .
$$

Then, from (4.4),

$$
\frac{\left\|U_{n-1}^{-1}\right\|_{2}\|A\|_{F}}{\|L\|_{F}} \leq \kappa_{L}(P A Q) \leq \kappa_{L}^{\prime}(P A Q)
$$

From (4.1) and (4.8), it follows that

$$
\frac{\kappa_{L}(P A Q)}{\kappa_{L}^{\prime}(P A Q)} \leq \frac{\left\|L^{-1}\right\|_{2}\left\|U^{-1}\right\|_{2}\|A\|_{F}}{\left(\left(\left\|U_{n-1}^{-1}\right\|_{2}\|A\|_{F}\right) /\|L\|_{F}\right)}=\frac{\left\|L^{-1}\right\|_{2}\left\|U^{-1}\right\|_{2}\|L\|_{F}}{\left\|U_{n-1}^{-1}\right\|_{2}} .
$$

On the other hand, (4.4) and (4.5) together give

$$
\frac{\kappa_{U}(P A Q)}{\kappa_{U}^{\prime}(P A Q)} \geq \frac{1}{\kappa_{2}(\tilde{U})} .
$$

Then, from (4.4),

$$
\frac{\left\|L^{-1}\right\|_{2}\|A\|_{F}}{\|U\|_{F}} \leq \kappa_{U}(P A Q) \leq \kappa_{U}^{\prime}(P A Q)
$$


Finally, from (4.1) and (4.9), it follows that

$$
\frac{\kappa_{U}(P A Q)}{\kappa_{U}^{\prime}(P A Q)} \leq \frac{\left\|L^{-1}\right\|_{2}\left\|U^{-1}\right\|_{2}\|A\|_{F}}{\left(\left(\left\|L^{-1}\right\|_{2}\|A\|_{F}\right) /\|U\|_{F}\right)}=\left\|U^{-1}\right\|_{2}\|U\|_{F} .
$$

\section{Perturbation theorem for the $Q R$ factors}

It is well known that $A+t G$ has a unique $\mathrm{QR}$ factorization with $\left\|A^{-1}\right\|_{2}\|G\|_{2} \epsilon$ $<1$ [9]. In this section, we present results on how $Q$ and $R$ change as $A$ changes, and prove a perturbation theorem for the $\mathrm{QR}$ factors.

THEOREM 5.1. Let $A \in \mathbb{R}^{n \times n}$ be nonsingular, and let $\Delta A \in \mathbb{R}^{n \times n}$ satisfy $\Delta A=\epsilon G$ and

$$
\left\|A^{-1}\right\|_{2}\|G\|_{2} \epsilon<1
$$

Suppose that

$$
\frac{1}{2}\|\dot{Q}(0)\|_{2} \epsilon<1 \quad \text { and } \quad \frac{1}{2}\left\|R^{-1} \dot{R}(0) \epsilon\right\|_{2}<1
$$

Then

$$
\begin{aligned}
\frac{\|\dot{R}(0)\|_{2}}{\|R\|_{2}} \leq \frac{\|G\|_{2}+(1+\sqrt{2})\left(\frac{1}{2}\|G\|_{2}\left\|A^{-1}\right\|_{2} \epsilon+\kappa_{2}(A)\right)\|\dot{A}(0)\|_{F}+O(\epsilon)}{\|A\|_{2}-\frac{1}{2}(1+\sqrt{2}) \kappa_{2}(A)\|\dot{A}(0)\|_{F} \epsilon}, \\
\|\dot{Q}(0)\|_{2} \leq \frac{\|G\|_{2}\left\|A^{-1}\right\|_{2}+\sqrt{2}\left(\frac{1}{2}\|G\|_{2}\left\|A^{-1}\right\|_{2}^{2} \epsilon+\left\|A^{-1}\right\|_{2}\right) \kappa_{2}(A)\|\dot{A}(0)\|_{F}+O(\epsilon)}{1-\frac{1}{2} \sqrt{2} \kappa_{2}(A)\left\|A^{-1}\right\|_{2}\|\dot{A}(0)\|_{F} \epsilon} .
\end{aligned}
$$

PROOF. From the assumptions, we deduce from [9] that

$$
A(t)=Q(t) R(t)
$$

where $Q(t)^{H} Q(t)=I$ and $R(t)$ is an upper triangular matrix with positive diagonal elements. Since $Q(t)$ and $R(t)$ are continuously differentiable for $|t| \leq \epsilon$ by a standard algorithm for QR factorization, it follows that

$$
Q(t)=Q(0)+\dot{Q}(0) t+O\left(\epsilon^{2}\right), \quad R(t)=R(0)+\dot{R}(0) t+O\left(\epsilon^{2}\right),
$$

where $Q(0)=Q, R(0)=R, Q(\epsilon)=Q+\Delta Q$ and $R(\epsilon)=R+\Delta R$. Setting $t=\epsilon$ and combining (5.1) with (5.2), we obtain

$$
\begin{aligned}
A+\epsilon G & =\left(Q+\dot{Q}(0) \epsilon+O\left(\epsilon^{2}\right)\right)\left(R+\dot{R}(0) \epsilon+O\left(\epsilon^{2}\right)\right) \\
& =Q R+Q \dot{R}(0) \epsilon+\dot{Q}(0) R \epsilon+\dot{Q}(0) \dot{R}(0) \epsilon^{2}+O\left(\epsilon^{2}\right) .
\end{aligned}
$$

Then

$$
\begin{aligned}
G & =Q \dot{R}(0)+\dot{Q}(0) R+\dot{Q}(0) \dot{R}(0) \epsilon+O(\epsilon) \\
& =\left(Q+\frac{1}{2} \dot{Q}(0) \epsilon\right) \dot{R}(0)+\dot{Q}(0)\left(R+\frac{1}{2} \dot{R}(0) \epsilon\right)+O(\epsilon)
\end{aligned}
$$


By setting $\bar{Q}=Q+\frac{1}{2} \dot{Q}(0) \epsilon, \bar{R}=R+\frac{1}{2} \dot{R}(0) \epsilon$ and $\bar{G}=G+O(\epsilon)$, we can write

$$
\bar{G}=\bar{Q} \dot{R}(0)+\dot{Q}(0) \bar{R},
$$

and hence

$$
\begin{aligned}
\dot{R}(0)= & \bar{Q}^{-1} \bar{G}-\bar{Q}^{-1} \dot{Q}(0) \bar{R} \\
= & \left(Q^{H}-\frac{1}{2} Q^{H} \dot{Q}(0) Q^{H} \epsilon+O\left(\epsilon^{2}\right)\right)(G+O(\epsilon)) \\
& -\left(Q^{H}-\frac{1}{2} Q^{H} \dot{Q}(0) Q^{H} \epsilon+O\left(\epsilon^{2}\right)\right) \dot{Q}(0)\left(R+\frac{1}{2} \dot{R}(0) \epsilon\right) \\
= & Q^{H} G-\frac{1}{2} Q^{H} \dot{Q}(0) Q^{H} G \epsilon-Q^{H} \dot{Q}(0) R-\frac{1}{2} Q^{H} \dot{Q}(0) \dot{R}(0) \epsilon+O(\epsilon) .
\end{aligned}
$$

Taking the 2-norm gives

$$
\begin{aligned}
\|\dot{R}(0)\|_{2} \leq & \|G\|_{2}+\frac{1}{2}\|\dot{Q}(0)\|_{2}\|G\|_{2} \epsilon+\|\dot{Q}(0)\|_{2}\|R\|_{2} \\
& +\frac{1}{2}\|\dot{Q}(0)\|_{2}\|\dot{R}(0)\|_{2} \epsilon+O(\epsilon) .
\end{aligned}
$$

Rearranging (5.4) yields

$$
\|\dot{R}(0)\|_{2} \leq \frac{\|G\|_{2}+\left(\frac{1}{2}\|G\|_{2} \epsilon+\|R\|_{2}\right)\|\dot{Q}(0)\|_{2}+O(\epsilon)}{1-\frac{1}{2}\|\dot{Q}(0)\|_{2} \epsilon} .
$$

From [9, Theorem 1.5], we obtain

$$
\|\dot{Q}(0)\|_{2} \leq\|\dot{Q}(0)\|_{F} \leq(1+\sqrt{2}) \kappa_{2}(A) \frac{\|\dot{A}(0)\|_{F}}{\|A\|_{2}} .
$$

Combining (5.5) with (5.6) and using $\|R\|_{2}=\|A\|_{2}$, we find that

$$
\frac{\|\dot{R}(0)\|_{2}}{\|R\|_{2}} \leq \frac{\|G\|_{2}+(1+\sqrt{2})\left(\frac{1}{2}\|G\|_{2}\left\|A^{-1}\right\|_{2} \epsilon+\kappa_{2}(A)\right)\|\dot{A}(0)\|_{F}+O(\epsilon)}{\|A\|_{2}-((1+\sqrt{2}) / 2) \kappa_{2}(A)\|\dot{A}(0)\|_{F} \epsilon} .
$$

On the other hand, we have from (5.3) that

$$
\begin{aligned}
\dot{Q}(0)= & \bar{G} \bar{R}^{-1}-\bar{Q} \dot{R}(0) \bar{R}^{-1} \\
= & (G+O(\epsilon))\left(R^{-1}-\frac{1}{2} R^{-1} \dot{R}(0) R^{-1} \epsilon+O\left(\epsilon^{2}\right)\right) \\
& -\left(Q+\frac{1}{2} \dot{Q}(0) \epsilon\right) \dot{R}(0)\left(R^{-1}-\frac{1}{2} R^{-1} \dot{R}(0) R^{-1} \epsilon+O\left(\epsilon^{2}\right)\right) \\
= & G R^{-1}-\frac{1}{2} G R^{-1} \dot{R}(0) R^{-1} \epsilon-Q \dot{R}(0) R^{-1}-\frac{1}{2} \dot{Q}(0) \dot{R}(0) R^{-1} \epsilon+O(\epsilon) .
\end{aligned}
$$

Taking the 2-norm and rearranging yields

$$
\|\dot{Q}(0)\|_{2} \leq \frac{\|G\|_{2}\left\|R^{-1}\right\|_{2}+\left(\frac{1}{2}\|G\|_{2}\left\|R^{-1}\right\|_{2}^{2} \epsilon+\left\|R^{-1}\right\|_{2}\right)\|\dot{R}(0)\|_{2}+O(\epsilon)}{1-\frac{1}{2}\|\dot{R}(0)\|_{2}\left\|R^{-1}\right\|_{2} \epsilon} .
$$

Similarly,

$$
\|\dot{R}(0)\|_{2} \leq\|\dot{R}(0)\|_{F} \leq \sqrt{2} \kappa_{2}(A)\|\dot{A}(0)\|_{F}
$$


from [9, Theorem 1.5]. Combining (5.7) with (5.8) and using $\left\|R^{-1}\right\|_{2}=\left\|A^{-1}\right\|_{2}$, we then obtain

$$
\begin{aligned}
& \|\dot{Q}(0)\|_{2} \\
& \leq \frac{\|G\|_{2}\left\|A^{-1}\right\|_{2}+\sqrt{2}\left(\frac{1}{2}\|G\|_{2}\left\|A^{-1}\right\|_{2}^{2} \epsilon+\left\|A^{-1}\right\|_{2}\right) \kappa_{2}(A)\|\dot{A}(0)\|_{F}+O(\epsilon)}{1-(\sqrt{2} / 2) \kappa_{2}(A)\left\|A^{-1}\right\|_{2}\|\dot{A}(0)\|_{F} \epsilon} .
\end{aligned}
$$

\section{Acknowledgements}

The authors would like to thank the anonymous referee and the editor Dr Emma Hunt very much for their helpful comments and suggestions that have helped to improve the presentation of this paper.

This research was supported by the 973 Program (2008CB317110), the NSFC (10771030), the Scientific and Technological Key Project of the Chinese Education Ministry (107098), the PhD Programs Fund of Chinese Universities (20070614001), the Sichuan Province Project for Applied Basic Research (2008JY0052), and the Project for Academic Leader and Group of UESTC.

\section{References}

[1] A. Barrland, "Perturbation bounds for the $L D L^{H}$ and the LU factorizations", BIT 31 (1991) $358-363$.

[2] X.-W. Chang and C. C. Paige, "On the sensitivity of the LU factorization”, BIT 38 (1998) 486-501.

[3] X.-W. Chang, C. C. Paige and G. W. Stewart, "Perturbation analyses for the QR factorization", SIAM J. Matrix Anal. Appl. 18 (1997) 775-791.

[4] F. M. Dopico and J. M. Molera, "Perturbation theory of factorizations of LU type through series expansions", SIAM J. Matrix Anal. Appl. 27 (2005) 561-581.

[5] N. J. Higham, Accuracy and stability of numerical algorithms (SIAM, Philadelphia, PA, 1996).

[6] S. Singer and S. Singer, "Rounding-error perturbation bounds for the indefinite QR factorization", Linear Algebra Appl. 309 (2000) 103-119.

[7] G. W. Stewart, "On the perturbation of LU, Cholesky, and QR factorizations", SIAM J. Matrix Anal. Appl. 14 (1993) 1141-1145.

[8] G. W. Stewart, "On the perturbation of LU and Cholesky factors", IMA J. Numer. Anal. 17 (1997) $1-6$.

[9] J.-G. Sun, "Perturbation bounds for the Cholesky and QR factorizations", BIT 31 (1991) 341-352.

[10] J.-G. Sun, "On perturbation bounds for the QR factorization", Linear Algebra Appl. 215 (1995) 95-112. 